\title{
Management of aortic stenosis in pregnancy
}

\author{
A.J.K. Pecoraro \\ Division of Cardiology, Department of Medicine, University of \\ Stellenbosch and Tygerberg Academic Hospital, South Africa \\ Address for correspondence: \\ A.J.K. Pecoraro \\ Division of Cardiology \\ Department of Medicine \\ University of Stellenbosch and Tygerberg Academic Hospital \\ PO Box 19063 \\ Tygerberg \\ 7505 \\ South Africa \\ Email: \\ pecoraro@sun.ac.za
}

The correct management of aortic stenosis (AS) hinges on thorough clinical assessment and meticulous incorporation of all parameters of the echocardiographic assessment. In the pregnant patient, or patients planning pregnancy, this is even more important. The assessment of AS can be very simple, but more often than not it is extremely difficult with many caveats and it has aptly been described as an art.(I) Managing AS in pregnancy is even more of an art, filled with many difficulties but also great rewards if correct assessments are made and correct decisions are taken. The decisions should be the result of a multidisciplinary team effort with the patient, cardiologist, obstetrician, pediatrician and anesthetist all playing their part towards the optimal care of mother and baby.

AS impacting on pregnancy is mostly congenital in origin, with bicuspid aortic valve disease being the most common cause. ${ }^{(2-5)}$ Rheumatic AS is another important cause, especially in developing countries. ${ }^{(6)}$ The majority of rheumatic AS is accompanied by rheumatic mitral valve involvement, although rare cases of isolated rheumatic AS have been seen. Supra- and infra-valvular stenoses are less common and their assessment and treatment will not be discussed in this review. ${ }^{(2)}$

\section{ASSESSMENT OF SEVERITY}

Assessment of AS in pregnancy follows the same principles as assessment in patients that are not pregnant. Three fundamental questions must be answered:

I. Is the patient symptomatic?

2. Is the aortic valve stenosis severe?

3. Can we attribute the symptoms to the aortic valve disease?

\section{ABSTRACT}

Aortic stenosis in pregnancy is most commonly related to congenital or bicuspid aortic stenosis, which is associated with an aortopathy and ascending aortic dilatation. The management of AS in pregnancy is based on a few key principles. First and foremost the accurate assessment of the patient's symptoms and confirmation, via echocardiography, of the degree of AS is of the utmost importance. Exercise testing is a very handy adjunct to quantify symptoms. Based on these findings, an informed process of discussion between the patient and a multidisciplinary team will lead to the correct management. As a general rule, patients who are truly asymptomatic with normal left ventricular systolic function and normal aortic root are able to tolerate pregnancy with a low maternal and fetal risk.

SAHeart 2014;11:76-79

The most important part of the assessment, as in other fields of medicine, is the history. The presence of symptoms, especially dyspnea, determines the prognosis. Clinical signs of severe AS are similar for pregnant patients when compared with non-pregnant patients.

Echocardiographic assessment of AS (Table I) should include peak and mean gradients, effective aortic valve area (indexed for body surface area) and dimensionless velocity index $(\mathrm{DVI}){ }^{(7)}$ It should be remembered that during pregnancy the stroke volume and left ventricular outflow tract velocity time integral (LVOT VTI) increase, which would increase the gradient across the aortic valve without change to the aortic valve area. ${ }^{(8)}$ It is important to align the jet of AS correctly. An eccentric jet is common in AS secondary to bicuspid valves and thus the freestanding probe should always be used to eliminate the underestimation of the gradient. ${ }^{(4)}$ The most common error that causes overestimation of aortic valve severity is incorrect measurement of the left ventricular outflow tract (LVOT) diameter. An LVOT diameter of less than $17 \mathrm{~mm}$ should always raise concern as to an incorrect measurement. The DVI is very useful as a "check and balance" for this common mistake. A DVI of less than 0.25 is in keeping with severe AS. Other helpful parameters in the assessment of AS is the jet shape and time to peak (TTP). A more parabolic shape with a late peak (TTP more than $100 \mathrm{~ms}$ ) indicates severe AS. (1,3) $^{-1}$ 
Two other very important parts of the echocardiographic assessment is left ventricular systolic (and diastolic) function and aortic root size. It is important to accurately quantify systolic function as impairment significantly increases the risk of adverse events in pregnancy. Normal diastolic function with a non-dilated left atrium is typical of the asymptomatic patient. As a bicuspid aortic valve is the most common cause of AS in the pregnant population, the risk of an associated aortopathy, which in turn increases the risk of aortic dilatation and dissection, deserves careful attention. The pathology and histology is similar to Marfan syndrome and leads to fusiform dilatation of the ascending aorta. The degree of dilation does not correlate with the severity of the AS, some patients with only mild AS have severe aortic root dilatation. ${ }^{(5)}$ The theory of post stenotic dilatation has largely been abandoned. An aortic root measurement at any level of more than $50 \mathrm{~mm}$ is associated with a marked increase in the risk of aortic dissection and surgery prior to conception should be advocated. ${ }^{(2,3)}$

Exercise testing of patients with severe AS prior or during pregnancy is an important part of the evaluation and management of patients. ${ }^{(2,3)}$

\section{NATURAL HISTORY OF AS IN PREGNANCY}

Cardiac output increases by $25-50 \%$ by the 24th week of gestation. This is accompanied by a similar increase in blood volume. ${ }^{(4,8)}$ Patients with severe AS that become symptomatic are unable to meet the haemodynamic and metabolic demand of pregnancy due to the fixed left ventricular outflow tract obstruction. ${ }^{(4)}$ In South Africa we frequently deal with mitral stenosis (MS) during pregnancy and are well aware of the dire haemodynamic consequences during pregnancy. The generally more favorable view of AS in pregnancy compared to MS is due to the more straightforward haemodynamic challenge. Increased cardiac output increases the trans aortic gradient but the rise in pressure is borne by the hypertrophied left ventricle in systole and not the pulmonary veins in diastole (as is the case with MS). The increase in extracellular fluid and plasma volume may increase the diastolic volume with only minimal increase in LV filling pressure. Patients with severe AS are mostly preload sensitive and pregnancy is unlikely to cause a catastrophic decrease in filling pressure. The effect of reducing filling time is

TABLE I: Echocardiographic parameters of severe AS.(7)

\begin{tabular}{l|l}
\hline Mean gradient* & $>40 \mathrm{mmHg}$ \\
\hline Peak velocity* & $4 \mathrm{~m} / \mathrm{s}$ \\
\hline Aortic valve area & $<1 \mathrm{~cm}^{2}$ \\
\hline Aortic valve area index for BSA & $<0.6 \mathrm{~cm}^{2} / \mathrm{m}^{2}$ \\
\hline Dimensionless Velocity index (DVI) & 0.25 \\
\hline Time to peak & $>100 \mathrm{~ms}$ \\
\hline
\end{tabular}

"Patients with normal cardiac output/transvalvular flow. BSA = body surface index. much less pronounced than in MS, so that the inevitable increase in heart rate during pregnancy presents less of a problem. Thus the ability to predict the haemodynamic impact of pregnancy on AS is simpler than in patients with MS and offers fewer surprises during pregnancy. ${ }^{(5)}$ Most patients with mild and moderate AS with normal left ventricular ejection fraction (LVEF) and aortic root diameter have a favorable outcome with pregnancy. ${ }^{(3,5,8)}$ Symptoms and exercise testing predict the maternal risk with severe AS. Patients who are asymptomatic and remain asymptomatic during exercise stress testing should be able to sustain pregnancy with limited maternal risk. ${ }^{(3)}$ Previous studies of severe AS in pregnancy site a 17\% maternal mortality rate, but a recent report by Silverside and colleagues report no maternal deaths with congenital AS of whom 59\% of patients had severe AS. ${ }^{(6,8)}$ Cardiac complications (heart failure and arrhythmias) with severe AS were reported at a rate of $\pm 10 \%$, while cardiac complications in mild/moderate AS are rare. ${ }^{(6)}$ Severe AS is also associated with a higher incidence of preterm labor, intrauterine growth retardation and lower birth weight. ${ }^{(1,8)}$ The onset of symptoms in patients during pregnancy usually manifests around 20 weeks gestation when the haemodynamic load on the heart significantly increases. Onset of symptoms related to AS before this stage is a poor prognostic sign. Obstetric complications specific to AS have not been specifically investigated, but hypertension related disorders and preterm labor is more common in patients with moderate/severe AS. ${ }^{(3)}$ When patients remain asymptomatic during pregnancy, the final haemodynamic challenge occur during the time of delivery and immediate postoperative period.

\section{PRE PREGNANCY EVALUATION AND MANAGEMENT}

All patients should receive detailed counseling regarding potential risks and treatments before and during pregnancy. ${ }^{(3,8,9)}$ It is especially true for patients with a bicuspid aortic valve as fetal congenital heart disease occurs in $\pm 4 \%$ of women with congenital AS. Fetal ultrasound is suggested when the mother has a bicuspid aortic valve. Pregnancy itself and the aortopathy associated carry a well-documented risk of aortic dissection. The absolute risk of dissection is difficult to estimate, but currently a diameter of less than $45 \mathrm{~mm}$ is considered safe to proceed with pregnancy (risk of dissection equal to the general population). ${ }^{(5)}$

Patients with severe AS who are asymptomatic should tolerate pregnancy provided:

\section{Normal LV systolic function.}

Aortic root diameter less than $45 \mathrm{~mm}$.

- Complete exercise testing without symptoms or fall in blood pressure.

Severe LVH excluded (<16mm posterior wall thickness). ${ }^{(3)}$ 
However, although the maternal mortality is low $(<1 \%)$ the risk of maternal cardiac and fetal complications is higher than in patients with mild/moderate AS. ${ }^{(6)}$ Patients with moderate or mild AS tolerate pregnancy well. It is important to remember mild or moderate AS should not cause symptoms in nonpregnant patients. If symptoms are present in patients with mild or moderate stenosis critical review of the clinical and echocardiographic findings is mandatory.

Patients with severe AS who are symptomatic, have impaired left ventricular (LV) systolic function or an aortic root diameter of more than $50 \mathrm{~mm}$ should be considered for surgery prior to conception. ${ }^{(3,5)}$ The group of patients with an aortic diameter between $45 \mathrm{~mm}$ and $50 \mathrm{~mm}$ pose a difficult challenge and should be individualised. It is important to note that dilatation of the aorta to less than $45 \mathrm{~mm}$ is common and does not carry additional risk. Aortic dissection in patients with bicuspid aortic valve disease and aortic root less than $50 \mathrm{~mm}$ remains rare and a balanced opinion should be given to patients during pre pregnancy counseling.

\section{MANAGEMENT OF SEVERE AS DURING PREGNANCY}

Unfortunately we as clinicians are often confronted with patients already pregnant at time of first consultation. During the first trimester the same principles as the pre pregnancy evaluation apply. Patients who are asymptomatic and can complete an exercise test should be advised to continue pregnancy. Patients who are symptomatic or become symptomatic during the exercise test, have impaired LV systolic function or dilated aortic root should be counseled about risks and termination advised. ${ }^{(3,5)}$ It is important to remember that patients often feel tired during pregnancy, especially during the first trimester, thus objective evidence of dyspnea should be sought before making any recommendation. Patients who remain asymptomatic during the second and third trimester should be carefully followed and vaginal delivery with assisted second stage is advised.

Patients who become symptomatic during the second and third trimester pose a difficult challenge. A trial of medical therapy in the form of bed rest and diuretics is usually the initial step.

If medical therapy fails, the options are:

Termination of pregnancy if not viable.

Delivery of fetus.

Balloon valvotomy or valve replacement.

It is important to involve a multidisciplinary team including an obstetrician, neonatologist, anesthetist and cardiothoracic surgeon to advise on the most appropriate therapy. Patients with a viable fetus should generally be delivered before an intervention on the valve is attempted. In patients with a non- viable fetus the decision lies between termination of pregnancy or intervention on the aortic valve. If the valve is suitable for balloon dilatation, it is generally favored to surgery with a nonviable fetus. A mobile valve with minimal calcium and aortic regurgitation has a low risk for complications during balloon dilatation with favorable short-term outcome. ${ }^{(5,8)}$ It must be noted that balloon valvotomy can induce aortic regurgitation and recurrent stenosis is common within 6-12 months. ${ }^{(5)}$ Radiation exposure should be limited and appropriate shielding used. Patients with severe AS not suitable for balloon dilatation should be considered for surgery, although the risk of spontaneous termination and fetal sequelae is higher than with balloon dilatation. Maternal mortality during cardiopulmonary bypass is similar to that of a non pregnant patient, but fetal mortality as high as $30 \%$ has been reported. ${ }^{(6)}$ Fetal morbidity also remains high with late neurological impairment at 3-6\% the most commonly encountered problem. Cardiac surgery is thus only recommended if medical or interventional therapy fails and the mother's life is threatened.(3) If surgery is to be performed, the period between the 13th and 28th week is preferred. The reason for this is the high risk of fetal malformations with surgery in the first trimester and an increase in maternal complications during the third trimester. Improvement in neonatal care has lead to an improved survival of premature infants and thus delivery via caesarian section before aortic valve surgery should be considered from 26 weeks onwards. Corticosteroids should be administered 24 hours before surgery to augment fetal lung maturity if possible.

\section{MODE OF DELIVERY}

For patients with asymptomatic severe AS that have progressed through pregnancy the preferred mode of delivery is vaginal. Some groups favor vaginal delivery with early epidural and good pain management to attenuate the increase in catecholamine release and cardiac output during labour. ${ }^{(6)}$ Vaginal delivery is associated with less blood loss, infection, venous thrombosis and thromboembolic complications compared to caesarian section. It is important that every patient has an individualised delivery plan. There is no consensus on absolute contraindications to vaginal delivery. The decision to recommend a caesarian section should be based on obstetric indications and the anticipated cardiopulmonary tolerance of the patient during delivery. ${ }^{(2-5)}$ Patients with severe heart failure or dilated aortic root $(>50 \mathrm{~mm})$ should be delivered via caesarean section. ${ }^{(3)}$ Some centers advocate routine caesarian section for patients with severe AS although that is not currently supported by the guidelines. ${ }^{(3)}$ Several publications have noted a high rate of caesarian section in these patients with favorable outcomes. ${ }^{(4)}$ Currently we would recommend discussion by a multi disciplinary team, before decisions on the mode of delivery is taken. Careful monitoring during delivery and in the post partum period is of the utmost importance as autotransfusion leads to an increase in circulating volume. This frequently leads to symptoms in patients with obstructive 
lesions such as AS. Endocarditis prophylaxis is not indicated during vaginal or caesarian delivery. Anesthetic management of these patients must be individualised based on the anticipated effect of selected anesthetic technique on the cardiac performance. ${ }^{(10)}$

\section{POST DELIVERY CARE}

The bulk of the haemodynamic load on the heart occurs during contractions and the hours after delivery. Patients with severe AS should be carefully monitored for the first 24-48 hours after delivery in a high care unit, with invasive monitoring and immediate correction of haemodynamic problems. Important goals of management of this period include maintaining adequate systemic vascular resistance and normal heart rate. Adequate pain relief is of the utmost importance. ${ }^{(4)}$ Patients who remain asymptomatic during this period can be treated according to standard protocols. Patients who become symptomatic should be treated with diuretics and carefully monitored until the effect of auto-transfusion has subsided.

\section{CONCLUSION}

The management of AS in pregnancy is based on a few key principles. First and foremost the accurate assessment of the patient's symptoms and confirmation, via echocardiography, of the degree of AS is of utmost importance. Exercise testing is a very handy adjunct to quantify symptoms. Based on these findings, an informed process of discussion with the patient, obstetrician, anesthetist, cardiothoracic surgeon and neonatologist will ensure good decision making and accurate recommendations. As a general rule, patients who are truly asymptomatic with normal left ventricular systolic function and normal aortic root are able to tolerate pregnancy with a low maternal and fetal risk.

\section{Conflict of interest: none declared.}

\section{REFERENCES}

I. Rajani R, Hancock J, Chambers J. The art of assessing AS. Heart 2012; 98:14-22.

2. Elkayam U, Bitar F. Valvular Heart disease and pregnancy. JACC 2005; 2:223-230.

3. Regitz-Zagrosek V, Lundqvist C, Borghi C, et al. ESC Guidelines on the management of cardiovascular diseases during pregnancy. EHJ 2011:32; 3147-3197.

4. loscovich AM, Goldszmidt E, Fadeev AV, et al. Peripartum anesthetic management of patients with aortic valve stenosis: aretrospective study and literature review. Int J Obstet Anesth. 2009 Oct; 18(4):379-86.

5. Traill TA. Valvular heart disease and pregnancy. Cardiol Clin. 2012 Aug; 30(3):369-81.

6. Hatton R, Colman JM, Sermer M, et al. Cardiac risks and management of complications in pregnant women with congenital heart disease. Future Cardiol. 2012 Mar;8(2):3।5-27.

7. Vahanian A, Alfieri $O$, Andreotti $F$, et al. Guidelines on the management of Valvular heart disease. EHJ 2012:33;245I-2496.

8. Yap SC, Takkenberg J), Witsenburg M, et al. Aortic stenosis at young adult age. Expert Rev Cardiovasc Ther. 2005 Nov;3(6): 1087-98.

9. Friedman T, Mani A, Elefteriades JA. Bicuspid aortic valve: clinical approach and scientific review of a common clinical entity. Expert Rev Cardiovasc Ther. 2008 Feb;6(2):235-48.

10. Kuczkowski KM, van Zundert A. Anesthesia for pregnant women with Valvular heart disease: The state-of-the-art. J Anesth. 2007;2 I (2):252-7. 